Revue des patrimoines

\title{
La structuration de corps professionnels au service des monuments historiques
}

\author{
PROJET MÉMOLOI 2015-2016
}

La loi du 31 décembre 2013 sur les monuments historiques

Volume 2 - De 1913 au Code du patrimoine, une loi en évolution

The structuring of the professional bodies involved in historic monument protection and management

\section{Marie-Anne Sire}

\section{OpenEdition}

\section{Journals}

Édition électronique

URL : http://journals.openedition.org/insitu/18617

DOI : 10.4000/insitu. 18617

ISSN : 1630-7305

\section{Éditeur}

Ministère de la culture

Référence électronique

Marie-Anne Sire, "La structuration de corps professionnels au service des monuments historiques », In Situ [En ligne], 30 | 2016, mis en ligne le 22 novembre 2018, consulté le 21 avril 2019. URL : http:// journals.openedition.org/insitu/18617 ; DOI : 10.4000/insitu.18617

Ce document a été généré automatiquement le 21 avril 2019.

In Situ Revues des patrimoines est mis à disposition selon les termes de la licence Creative Commons Attribution - Pas d'Utilisation Commerciale - Pas de Modification 4.0 International. 


\section{La structuration de corps professionnels au service des monuments historiques}

PROJET MÉMOLOI 2015-2016

La loi du 31 décembre 2013 sur les monuments historiques

Volume 2 - De 1913 au Code du patrimoine, une loi en évolution

The structuring of the professional bodies involved in historic monument protection and management

Marie-Anne Sire

Cet article n'aurait pu exister sans les précieuses informations que m'ont communiquées $D$. Barraud, J.P. Ciret, L. Fournier, M. Gauthier, C. Hottin, J. Kagan, X. de Massary, M. Monnier, C. Oudin, M.-S. de Ponthaud, J. Prevot, F. Schoenstein, P. Thibaut, D. Watin-Granchamps. Qu'ils trouvent tous ici l'expression de ma reconnaissance.

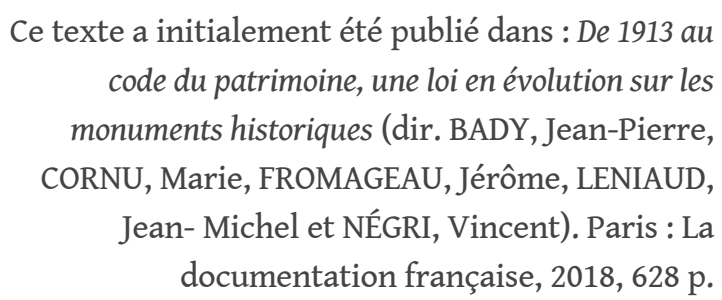

1 Par nature, la protection du patrimoine national - qui reflète l'héritage commun transmis d'une génération à l'autre - intéresse chacun d'entre nous. La reconnaissance de cet intérêt «public » qui fait «le monument historique » a relevé d'abord d'érudits isolés avant d'être revendiquée à la période révolutionnaire par les membres de l'Assemblée constituante comme une responsabilité de la Nation tout entière. Cette volonté politique a ouvert la voie à la professionnalisation progressive de ceux qui ont été appelés à organiser ce repérage et qui ont veillé ensuite à la sauvegarde des monuments et des objets jugés « historiques ». Celle-ci est apparue comme une nécessité au fur et à mesure que les exigences scientifiques, liées au travail d'inventaire et aux opérations de 
restauration, se sont affirmées. Il a fallu cette lente prise de conscience pour que les procédures mises en place prévoient de remplacer les précurseurs éclairés des sociétés savantes par des experts, ayant reçu une formation spécifique, et susceptibles d'engager leur responsabilité en cas de contentieux. Cette dynamique de professionnalisation a concerné à la fois les métiers de la conservation et de l'architecture, de la documentation, de la recherche et de la médiation, au sein de l'État comme, plus récemment, au sein des collectivités locales; pour les travaux de restauration, elle a touché à la fois les équipes qui assurent la maîtrise d'œuvre et celles qui sont responsables de la maîtrise d'ouvrage. Elle s'est articulée en grandes étapes, à des dates distinctes selon les métiers concernés, et qui sont associées à des textes législatifs ou règlementaires.

\section{La Surintendance des Bâtiments royaux}

2 Les premiers monuments, dont l'entretien est confié à une véritable administration, sont en France les bâtiments royaux. Ils sont confiés jusqu'à la fin du Xvi siècle aux trésoriers de France qui ont le pouvoir d'ordonner les réparations et les constructions nouvelles. Parmi ces bâtiments, les «maisons royales », où loge le souverain avec sa famille, relèvent directement de son autorité. Sous Louis XII et François $\mathrm{I}^{\mathrm{er}}$, chaque chantier dans ces édifices est confié à un commissaire qui a sous ses ordres un contrôleur et un payeur. Certains de ces commissaires, chargés de plusieurs édifices, prennent le titre de « superintendant» ou « surintendant ». Henri II et ses fils leur associent des architectes. Philibert Delorme, Le Primatice, Jean Bullant sont ainsi nommés surintendants. Sous Henri III, Baptiste Androuet Du Cerceau, puis son frère Jacques II, sont chargés de la superintendance du bâtiment neuf du Louvre.

Henri IV ${ }^{1}$ veille à dissocier les fonctions d'ordonnateur et d'architecte : les surintendants commandent les travaux, les architectes les exécutent. Sully devient surintendant en 1602 et à ce titre, est l'ordonnateur principal des travaux commandés par le roi au Louvre, aux Tuileries et à Saint-Germain-en-Laye. Il est alors déjà grand voyer de France et surintendant des fortifications et apprécié par le roi pour ses qualités de gestionnaire. En 1664, la mission de surintendant est confiée par Louis XIV à Jean-Baptiste Colbert, déjà contrôleur général des finances, associée à la charge de veiller sur les arts, les tapisseries et les manufactures. En 1699, la surintendance est à nouveau attribuée à un architecte, Jules-Hardouin Mansart, avant d'être rebaptisée « direction générale des Bâtiments » et de revenir à des conseillers proches du roi, le marquis de Marigny de 1751 à 1774 ou le comte d'Angiviller de 1774 à 1789 . Au début du XVIII ${ }^{\text {e }}$ siècle, le surintendant dispose d'une équipe de près de 150 personnes qui comprend des architectes, des intendants, des contrôleurs, des trésoriers et un historiographe.

\section{La période révolutionnaire : la naissance du métier de « conservateur»}

4 La confiscation des biens de l'Église en 1789, suivie en 1792 par celle des biens de la Couronne et des propriétés des nobles émigrés, donne à la Nation une responsabilité nouvelle: il faut choisir, parmi les monuments et les œuvres devenus propriétés publiques, ceux qui méritent d'être conservés et transmis aux générations futures. Pour y parvenir, l'Assemblée constituante prescrit le recensement et le tri des œuvres d'art ; les 
unes doivent être recueillies dans des musées, les autres sont promises à la vente ou à la fonte. La commission des Monuments, créée le 16 décembre 1790, réunit artistes et savants et élabore les premières préconisations. Le nouveau musée prévu au Louvre est appelé à recueillir tableaux et statues dans le but d'affirmer un héritage national et de " nourrir le goût des beaux arts ${ }^{2}$; les « brevets de commissaire du museum » détaillent le rôle des nouveaux " conservateurs ": travailler "à l'emplacement de tous les objets d'art, de manière que chaque objet soit vu dans son meilleur état ", inventer "un plan d'organisation" pour accueillir le public et notamment les artistes. Les personnes choisies sont généralement recrutées sur leurs talents de dessinateurs et non sur des compétences d'historien d'art.

Une attention toute particulière est accordée simultanément aux archives de l'Assemblée constituante ${ }^{3}$ afin de s'assurer de la transparence de ses décisions: un service des archives de l'Assemblée est créé le 29 juillet 1789 qui devient le service des Archives nationales le 12 septembre 1790. Il est appelé par la loi du 25 juin 1794 à recevoir l'ensemble des archives publiques et à former la tête d'un réseau formé par toutes les archives départementales. Pour veiller sur les bâtiments affectés à l'État, un conseil général des Bâtiments civils est créé en 1795 , au sein du ministère de l'Intérieur, associé à un corps d'architectes des bâtiments civils choisis parmi les anciens pensionnaires romains. Dans chaque département, un architecte assume l'entretien ou la construction des bâtiments départementaux.

\section{L'Empire et La Restauration : les premiers inventaires}

Sous l'Empire, l'idée d'inventorier les monuments et les œuvres qui intéressent l'histoire nationale est lancée par l'archéologue Alexandre de Laborde (1773-1842) ${ }^{4}$ qui inspire une circulaire transmise aux préfets par le comte de Montalivet, ministre de l'Intérieur, le 18 mai $1810^{5}$ : il leur demande de dresser la liste des châteaux, abbayes et tombeaux présents dans leur département, d'en définir l'intérêt architectural et de décrire leur localisation, leur état et leur entretien. Les rares réponses reçues montrent alors combien les préfets peinent à mobiliser les compétences locales sur ces domaines. Les circulaires suivantes qui relancent l'enquête sous la Restauration, les 23 mars 1817, puis 8 avril 1819, reçoivent des réponses de plus en plus nombreuses, montrant l'engagement des sociétés archéologiques locales. Les pillages et dépeçages commis par «la Bande noire » dans les années 1820 et la destruction d'une grande partie de l'abbaye de Cluny amènent Victor Hugo à déclarer en 1825 la " guerre aux démolisseurs ${ }^{6}$ en alertant l'opinion publique. Il réclame une loi pour protéger les monuments et des sanctions contre ceux qui les détruisent.

\section{Le « moment Guizot » sous la monarchie de Juillet : la création d'un poste d'inspecteur général et d'une commission nationale des Monuments historiques}

Devenu ministre de l'Intérieur de la toute jeune monarchie de Juillet, l'historien François Guizot (1787-1874) ${ }^{7}$ convaincra le roi Louis-Philippe, dans son célèbre rapport du 21 octobre 1830, de la nécessité de créer un poste d'inspecteur général des monuments 
historiques chargé de veiller à sauvegarder et à faire connaître «l'admirable enchaînement de nos antiquités nationales ». Il aura pour tâche de

parcourir successivement les départements de la France, s'assurer sur les lieux de l'importance historique ou du mérite d'art des monuments, recueillir tous les renseignements qui se rapportent à la dispersion des titres ou des objetsaccessoires qui peuvent éclairer sur l'origine, les progrès de la destruction de chaque édifice (...), éclairer les propriétaires et les détenteurs sur l'intérêt de leurs soins et stimuler enfin en les dirigeant le rôle de tous les conseils du département et de municipalité de manière à ce qu'aucun monument d'un mérite incontestable ne périsse par cause d'ignorance et de précipitation (...), de manière aussi à ce que la bonne volonté des autorités ou des particuliers ne s'épuise pas sur des objets indignes de leurs soins ${ }^{8}$.

Le premier titulaire de ce poste fut un jeune juriste de 28 ans, Ludovic Vitet (1802-1873) proche de Guizot et de Casimir Périer et, par ses ascendances normandes, d'Auguste Le Prévost (1787-1859) ${ }^{10}$, très actif au sein de la toute jeune Société des antiquaires de Normandie $^{11}$. Il quitte ses fonctions pour devenir député de Seine-Inférieure en 1834 et Thiers choisit, pour le remplacer, Prosper Mérimée ${ }^{12}$. Comme Vitet, celui-ci n'a aucune formation archéologique ; il est alors chef de cabinet du comte d'Argout au ministère du Commerce et des Travaux publics. Il a une formation classique et quelques rudiments de droit. Par son père, Léonor Mérimée, secrétaire général de l'École des beaux-arts, il est familier des milieux artistiques et a publié à cette date deux ouvrages : La Jacquerie (Paris, Brissot-Thivars, 1828) et Chronique du temps de Charles IX (Paris, Mesnier, 1829).

Pour l'aider à hiérarchiser les priorités et répartir les subventions de l'État, Montalivet crée en 1837 la commission des Monuments historiques; il y nomme des archéologues comme Auguste Le Prévost, le baron Taylor (1789-1879), deux architectes, Auguste Caristie (1783-1862) 13 - qui a restauré, entre autres, l'arc et le théâtre antique d'Orange et Félix Duban (1797-1854) ${ }^{14}$ - qui s'était déclaré avec Labrouste contre Quatremère de Quincy et la tradition académique, deux députés, Alphonse Denis ${ }^{15}$ et Philippe de Golbéry 16 - connus pour leurs publications savantes - ainsi que Charles Lenormant (1802-1859) ${ }^{17}$, conservateur des Monnaies et Médailles de la Bibliothèque nationale. La commission nouvellement créée examine le résultat de la nouvelle enquête lancée auprès des préfets le 10 août 1837 ; celle-ci leur demandait d'établir une liste des monuments anciens à conserver dans leur département, de les «classer » par ordre d'importance et d'estimer les sommes nécessaires à leur restauration. Elle tente de s'affranchir de la tutelle du prestigieux conseil général des Bâtiments civils qui a hérité des attributions de l'ancienne surintendance des Bâtiments du roi et qui est chargé des monuments appartenant à l'État, à l'exception des cathédrales, qui relèvent de l'administration des Cultes. Elle doit aussi travailler en liaison avec le comité des Arts et Monuments, section du Comité pour la recherche et la publication des documents inédits relatifs à l'histoire de France créé en 1834 par François Guizot, pour travailler à la publication des textes anciens inédits ${ }^{18}$. On y retrouve Mérimée, Vitet, Le Prévost et Lenormant, mais aussi Victor Hugo, Cousin, Albert Lenoir et Didron.

L'entretien des édifices diocésains est alors du ressort d'architectes départementaux, vers lesquels la commission se tourne pour demander les premiers devis de travaux concernant les urgences signalées. Un corps d' "architectes diocésains " ${ }^{19}$ est créé en 1848 : ils sont chargés des travaux sur les cathédrales, les évêchés et les séminaires. Leurs chantiers sont suivis localement par un «inspecteur des travaux diocésains » et ils agissent sous l'autorité d'inspecteurs généraux diocésains dont Viollet-le-Duc fait partie 
de 1853 à 1874. Ce dernier est en même temps « attaché à la commission des Monuments historiques » et appelé dès 1840 à restaurer la basilique de Vézelay. Ce choix ne manque pas d'audace : Viollet-le-Duc n'a pas suivi la voie habituelle de l'École des beaux-arts, qui privilégie la théorie et le relevé de bâtiments et prend pour modèle l'architecture antique; il a jugé peu adaptée cette approche, qui forme les architectes des bâtiments civils, pour affronter les problèmes liés à la consolidation ou à la restauration d'un édifice médiéval. Sa formation s'est faite au contact du terrain, ce qui est inédit.

\section{Le Second Empire : le développement du service des Monuments historiques face aux administrations des Cultes et des Bâtiments civils et Palais nationaux}

11 Sous le Second Empire, le service naissant des Monuments historiques est rattaché à la Maison de l'Empereur. Mérimée s'efforce de lui donner pertinence et efficacité à la fois dans les choix de protection et dans les partis de restauration envisagés. Viollet-le-Duc lui fournit le socle théorique qui manquait aux premiers débats en commission : il publie de 1854 à 1868 son Dictionnaire raisonné de l'architecture française du XI ${ }^{e} a u \mathrm{XVI}^{e}$ siècle $e^{20}$, dans lequel il indique notamment, à l'article "Restauration ", que restaurer un édifice signifie pour lui « le rétablir dans un état qui peut n'avoir jamais existé ». Il y développe aussi son principe d'unité de style qui implique que chaque monument soit restauré « dans le style qui lui appartient ", charge à l'architecte d'identifier ce style et d'éliminer les ajouts tardifs qui en polluent la lecture. Les architectes appelés à restaurer les monuments repérés par la commission sont cooptés et font partie de ses élèves ou de ceux d'Émile Boeswillwald (1815-1896) ${ }^{21}$.

\section{Les années 1880-1890: la fin de la cooptation et le recrutement par concours des architectes en chef des monuments historiques}

12 Ce système de cooptation est remis en question dans le dernier quart du XIX ${ }^{e}$ siècle et on assiste alors à la mise au point d'un modèle de formation théorique pour un recrutement des architectes par concours ${ }^{22}$. Un premier concours est organisé en 1884 pour recruter des architectes diocésains. Dix ans plus tard est mis en place le premier concours de recrutement des architectes en chef des monuments historiques. Les candidats retenus ne sont pas tenus d'habiter leur département et ont à leurs côtés les architectes ordinaires mis en place par la loi du 30 mars 1887 dans chaque département, chargés de l'entretien ordinaire des monuments historiques. L'organisation de ces concours est rendue possible par la création de deux nouvelles filières de formation : la première, en 1887, au musée de Sculpture comparée, au palais du Trocadéro, avec le cours d'Anatole de Baudot (1834-1915) ${ }^{23}$, adjoint de Viollet-le-Duc, qui est ouvert par arrêté conjoint de la direction des Cultes et de la direction des Beaux-Arts pour «former le personnel dont l'État a besoin pour la restauration des édifices diocésains et des monuments historiques ». C'est la naissance de l'École de Chaillot. La seconde filière est ouverte en 1892, à l'École des beaux-arts où une nouvelle "chaire d'architecture française du Moyen Âge et de la Renaissance » est confiée à Paul Boeswillwald (1844-1931) ${ }^{24}$. Ces décisions sont prises en accompagnement de la loi du 30 mars 1887 qui marque la première étape législative pour 
donner au « classement » des immeubles et des objets un socle juridique. Simultanément, l'inspection générale des monuments historiques est réorganisée et un poste d'inspecteur général adjoint chargé des objets mobiliers est créé le 30 avril 1893 et confié à Paul-Frantz Marcou $^{25}$.

\section{Les années 1905-1913: la création des nouveaux métiers liés à la protection des objets mobiliers}

13 La loi du 9 décembre 1905 concernant la "Séparation des Églises et de l'État» s'accompagne du transfert des édifices de culte classés de l'administration des Cultes à celles des Beaux-Arts, ce qui confie désormais au service des Monuments historiques le soin d'assurer l'entretien et la restauration de l'ensemble des monuments présentant un intérêt national, selon des critères uniquement artistiques et quelle que soit leur affectation. La loi impose aussi de nombreux classements complémentaires pour des édifices religieux jugés en péril. Deux décrets réorganisent le 12 avril 1907 le service des Monuments historiques, le premier fixant le cadre général et le second précisant l'organisation du personnel technique. Celui-ci relève directement du ministre et comprend des architectes en chef, des architectes ordinaires, des vérificateurs et des gardiens de monuments. Le nombre des architectes en chef des monuments historiques ne peut dépasser 40. Ils sont recrutés par concours et rémunérés par « honoraires ». Les architectes ordinaires, un par département, les secondent. Ils sont choisis parmi les architectes qui exercent dans leur département et sont nommés pour quatre ans. Les architectes diocésains continuent à assurer la conservation des édifices cultuels dont ils étaient chargés. Leurs emplois sont supprimés par voie d'extinction.

14 La loi prévoit aussi, dans son article 16, que tous les objets mobiliers qui n'ont pas encore été classés le soient en bloc, avec classement définitif dans un délai de trois ans pour ceux « dont la conservation présenterait, au point de vue de l'histoire ou de l'art, un intérêt suffisant ». Pour répondre à cette nouvelle urgence, il est créé dans chaque département une commission réunissant l'archiviste et l'architecte départementaux, l'architecte diocésain, l'architecte ordinaire des monuments historiques et les représentants de sociétés archéologiques locales. Cette commission doit signaler à l'administration centrale les objets non encore répertoriés qui auraient un intérêt véritable au point de vue de l'histoire ou de l'art. Il est aussi créé un poste de « conservateur des antiquités et objets d'art » qui est chargé du « travail de classement et de la surveillance des antiquités et objets d'art et autres objets visés par la loi du 30 mars 1887 ». Ces conservateurs départementaux sont choisis parmi les personnes possédant une compétence reconnue en matière d'art, d'archéologie ou d'histoire et qui résident dans le département. Ils travaillent sous le contrôle de trois inspecteurs généraux adjoints des antiquités et objets d'art et de six inspecteurs. Ils sont «choisis parmi les anciens membres des Écoles françaises d'Athènes et de Rome, les anciens élèves diplômés de l'École du Louvre, les archivistes paléographes, les fonctionnaires appartenant à l'administration des BeauxArts » et nommés par arrêté ministériel après consultation du comité consultatif des inspecteurs généraux.

15 Une nouvelle loi est votée le 31 décembre 1913 et vient renforcer les pouvoirs de l'État en matière de protection des monuments. Elle crée les conditions favorables à la poursuite de la professionnalisation en cours des métiers d'architecte et d'inspecteur des 
monuments historiques. La loi du 10 juillet 1914 institue en outre une Caisse nationale des monuments historiques et préhistoriques qui a pour but de réunir des fonds destinés à acquérir des monuments déjà classés ou des immeubles en instance de classement et à y financer des travaux d'entretien et de restauration.

\section{L'entre-deux-guerres : les décrets inspirés par Paul Léon pour professionnaliser l'inspection et les architectes départementaux}

Les années d'entre-deux-guerres sont marquées par la personnalité remarquable de Paul Léon, directeur du service, qui va réexaminer le fonctionnement du service des Monuments historiques pour l'adapter au nombre croissant de monuments protégés ${ }^{26}$. Il est à l'origine, en 1935, de deux décrets importants pour la professionnalisation des métiers : le premier, daté du 11 mai 1935, concerne l'organisation de l'inspection générale et de l'inspection des monuments historiques : il prévoit sept inspecteurs généraux dont quatre chargés de la conservation des édifices classés - qui ne peuvent être simultanément chargés de travaux de restauration - un inspecteur général des sites, un autre de la conservation des antiquités et objets d'art et un de l'examen des affaires contentieuses. Ils sont tous nommés pour 5 ans renouvelables. Les inspecteurs des monuments historiques sont désormais recrutés par concours pour assister l'inspecteur général chargé des antiquités et objets d'art et encadrent l'activité des conservateurs des antiquités et objets d'art, présents dans chaque département.

Le second décret date du 20 décembre 1935 et redéfinit la fonction des architectes ordinaires qui deviennent architectes des monuments historiques et sont désormais recrutés sur concours par examen suivi d'un stage probatoire ouvert aux candidats ayant entre 28 et 45 ans. Ils sont rémunérés au moyen d'honoraires et de vacations et soumis à l'autorité hiérarchique des architectes en chef et de l'administration.

18 Le cours du Trocadéro ${ }^{27}$, interrompu par la guerre et la mort d'Anatole de Baudot, reprend avec un éventail de cours plus riche qui inclut désormais, en plus du cours sur la reconstruction et les charpentes, des cours sur l'architecture civile et militaire, la sculpture, le vitrail et le mobilier.

19 L'apparition en 1927 d'un premier niveau de protection pour les immeubles, ne requérant qu'un « intérêt suffisant » au lieu d'un « intérêt public », élargit le champ des édifices qui peuvent espérer une protection. La loi Cornudet sur les plans d'extension et d'aménagement des villes, en 1919, puis la loi du 2 mai 1930 sur les sites complètent le dispositif en prévoyant la planification urbaine et la protection des paysages.

\section{Les années de guerre, 1940-1945 : les lois sur les fouilles archéologiques et les abords}

L'archéologie française s'est développée dans la seconde moitié du XIX siècle, plus rapidement hors des frontières, notamment dans les territoires qui ont connu une présence coloniale, que dans l'Hexagone, où elle relève encore largement des sociétés savantes. Le service des Missions, créé en 1842, regroupe les commissions d'Afrique du Nord et d'Algérie, d'Afghanistan, d'Asie mineure et continentale, du Caire, de Perse, et 
d'Albanie. Afin de s'inspirer des expériences acquises dans ces missions internationales et d'instituer un contrôle sur les fouilles entreprises sur le sol national, une loi est promulguée le 27 septembre 1941 portant réglementation des fouilles archéologiques: elle oblige à demander une autorisation pour fouiller, à déclarer toute découverte fortuite et permet à l'État d'entreprendre lui-même des fouilles. Elle est complétée le 21 janvier 1942 par une autre loi qui confie au Centre national de la recherche scientifique (CNRS), créé en 1939, la direction scientifique des travaux et subventions et des publications et qui prévoit un maillage du territoire en circonscriptions, six en "préhistoire » et quinze en " histoire ", c'est-à-dire " antiquités celtiques, grecques et gallo-romaines ». Chaque circonscription est confiée à un spécialiste appelé "directeur des antiquités", choisi parmi les membres de l'Institut, les anciens élèves des Écoles françaises d'Athènes ou de Rome, les professeurs des universités, les conservateurs de musées, les archivistes. Ils reçoivent une simple indemnité. La loi de 1941 est validée en 1945 ; par contre celle de 1942 est modifiée et la coordination scientifique est transférée du CNRS à la direction de l'Architecture ${ }^{28}$.

21 Cet effort pour organiser un contrôle des fouilles s'accompagne d'une prise en compte des abords des monuments historiques par la loi du 25 février 1943, qui définit - autour de chaque édifice classé ou inscrit - un périmètre de 500 mètres dans lequel tout projet doit obtenir l'accord de l'architecte départemental.

\section{L'après-guerre, 1945-1959 : le renforcement de la professionnalisation et le nouveau maillage du territoire}

Les années d'après-guerre marquent une étape décisive dans la professionnalisation des architectes chargés de l'entretien des monuments historiques avec la création par le décret du 21 février 1946 d'un nouveau corps de fonctionnaires, les architectes des bâtiments de France ${ }^{29}$. Ils sont appelés à agir, dans chaque département, au sein d'une agence dite "des Bâtiments de France ", placée sous leur autorité. Leurs missions sont plus larges que celles des anciens architectes ordinaires: ils deviennent les correspondants permanents de l'État dans les départements pour le suivi du contrôle et de l'entretien des monuments historiques, mais également dans le domaine des sites, perspectives et paysages et veillent sur l'application des lois. Ils sont aussi chargés des réparations ordinaires dans les bâtiments civils et palais nationaux de leurs départements. La répartition des responsabilités entre eux et les architectes en chef des monuments historiques se clarifie : à eux, la maîtrise d'œuvre des travaux d'entretien sur les monuments historiques, aux architectes en chef, les travaux de gros œuvre et les travaux de restauration.

Les agences des Bâtiments de France sont rebaptisées en 1978 « services départementaux de l'Architecture et du Patrimoine " puis « services territoriaux de l'Architecture et du Patrimoine » en 2010 quand ils sont intégrés au sein des directions régionales des Affaires culturelles et unités départementales de l'architecture et du patrimoine depuis 2015. 


\section{La période Malraux : le lancement des secteurs sauvegardés et de l'Inventaire général} pour que soient classés au titre des monuments historiques plusieurs édifices emblématiques du xx $\mathrm{x}^{\mathrm{e}}$ siècle, dus à Le Corbusier, aux frères Perret ou à Eugène Beaudoin et Marcel Lods. En lançant en 1964 avec André Chastel « l'Inventaire général des richesses artistiques de la France $»^{30}$, André Malraux ouvre la réflexion sur le patrimoine à des pans entiers de la mémoire jusque-là méconnus, et qui méritent pourtant d'y être intégrés : le patrimoine rural, comme les bâtiments à vocation scientifique, industrielle ou technique concentrent l'attention des nouveaux «conservateurs de l'Inventaire général», qui plaident pour leur protection au titre des monuments historiques en construisant le cadre scientifique nécessaire fondé sur la méthodologie et le vocabulaire appropriés ${ }^{31}$. Le nouveau ministre prend aussi la tutelle du bureau des Fouilles et des Antiquités et met en place, le 23 avril 1964, le conseil supérieur de la Recherche archéologique. En parallèle, il établit des comités régionaux des Affaires culturelles, appelés à s'appuyer sur les conservations régionales des Bâtiments de France déjà existantes pour coordonner les projets culturels.

\section{«L'explosion patrimoniale », 1970-2000 : la mise en place des directions régionales des Affaires culturelles, la déconcentration de la protection, le nouveau corps unifié des conservateurs du patrimoine et l'apparition des études préalables}

Un décret du 3 février $1977^{32}$ institue dans chacune des vingt-deux régions une direction régionale des Affaires culturelles (DRAC) ; un autre décret du 12 mai 1981 met en place, au sein de chacune d'entre elles, une " conservation régionale des monuments historiques » qui abrite une "cellule de recensement » composée de chargés d'études documentaires appelés à constituer les dossiers de protection des immeubles et une cellule «travaux et marchés ", composée d'ingénieurs et de techniciens des services culturels, qui exercent alors la maîtrise d'ouvrage sur les travaux de restauration engagés sur les monuments appartenant à l'État comme sur la plupart des monuments historiques ne lui appartenant pas. Les conservateurs régionaux des monuments historiques sont recrutés parmi les architectes des bâtiments de France, les inspecteurs des monuments historiques ou les attachés des services extérieurs.

Simultanément, le recrutement de chercheurs pour passer du préinventaire à l'inventaire, en respectant un vocabulaire et une méthodologie communs, se met 
progressivement en place : ils sont recrutés par concours interne en 1979, et par concours externe à partir de 1982 avec les conservateurs de l'Archéologie. Les commissions d'Inventaire et des conservateurs de l'Inventaire sont rattachées en 1985 aux directions régionales.

La même année, la procédure d'inscription sur l'inventaire supplémentaire est déconcentrée et il est institué une commission régionale pour le Patrimoine historique, archéologique et ethnologique (COREPHAE, rebaptisées commissions régionales pour le Patrimoine et les Sites ou CRPS en 1999) afin d'examiner les dossiers constitués par les " chargés de recensement "; ceux-ci sont recrutés par concours et intégrés à partir de 1978 au personnel de documentation du ministère. La dimension «ethnologique » du patrimoine à protéger s'inscrit dans le prolongement de la mise en place en $1980 \mathrm{du}$ conseil national pour le Patrimoine ethnologique et de la nomination de conseillers pour l'ethnologie dans chaque $\mathrm{DRAC}^{33}$. Pour des patrimoines spécifiques comme le patrimoine ferroviaire, maritime, ou le patrimoine campanaire, de nouveaux experts sont appelés à partir de 1985. Pour les orgues, des techniciens-conseils, chargés de la protection et du suivi des travaux, sont nommés en 1995.

Sous le double effet de la déconcentration de la procédure d'inscription et de l'élargissement des critères de protection, le nombre des monuments et objets inscrits augmente de manière accélérée. Pierre Nora évoque ce développement soudain comme une véritable « explosion patrimoniale $»^{34}$.

La complexité des approches en matière de restauration amène la création, en 1970, du Laboratoire de recherche des Monuments historiques (LRMH), qui est destiné à aider les maîtres d'œuvre et les maîtres d'ouvrage à réaliser les investigations nécessaires pour affiner le diagnostic sur les altérations et mieux définir les projets de travaux. La circulaire du 5 août 1985 relative à l'organisation des études et travaux sur les monuments historiques classés rend obligatoire cette démarche d'amélioration de la connaissance en amont du projet. L'objectif prioritaire est de mieux maîtriser les délais et les coûts. Dans les faits, les nouvelles études préalables iront bien au-delà : elles visent à réunir les éléments nécessaires pour établir le programme de l'opération en y incluant les diagnostics archéologiques nécessaires ainsi que les éventuelles études sur la nature des matériaux, des liants, la stratigraphie des polychromies... Chaque projet s'inscrit désormais dans une vision globale, à laquelle participent l'ensemble des services patrimoniaux : conservations régionales des Monuments historiques, services régionaux de l'Inventaire, de l'Archéologie, services départementaux de l'Architecture et du Patrimoine et, en administration centrale, au sein de la direction du Patrimoine créée en 1978, inspection, Laboratoire de recherche des monuments historiques, bibliothèque et archives des Monuments historiques.

31 L'accroissement du nombre des monuments protégés induit la modification par décret, le 20 novembre 1980, du statut d'architecte en chef des monuments historiques, inchangé depuis 1907 ; il supprime la règle qui limitait leur nombre à un maximum de 40 et indique que leur nombre évoluera en fonction des besoins du service. Il permet en outre aux propriétaires privés ou publics autres que l'État de choisir leur architecte.

Pendant cette période, les restaurateurs assistent eux aussi à une professionnalisation de leurs filières de formation: un Institut français de la restauration des œuvres d'art (IFROA) est mis en place en 1977 pour leur proposer un parcours plus adapté aux nouveaux principes de "conservation préventive » présentés dans la charte de Venise (1964). Certains de ces restaurateurs se portent candidats, au terme de leur formation, 
aux concours de "chef de travaux d'art» ou de «technicien d'art» organisés par le ministère.

Du côté des conservateurs, les cinq filières existantes (Musées, Inventaire, Archéologie, Archives et Monuments historiques) sont réunies au sein d'un nouveau corps de "conservateurs du patrimoine", créé par le décret du 16 mai 1990. Il prévoit un seul concours pour leur recrutement et met en place "l'École nationale du patrimoine ». L'IFROA lui est rattaché en 1996 et l'École, qui devient en 2001 «l'Institut national du patrimoine » (INP), forme désormais à la fois les conservateurs et les restaurateurs. Les conservateurs régionaux de l'Archéologie, qui ont remplacé en 1991 les directeurs des Antiquités, adaptent leurs services, au sein des directions régionales, au suivi des opérations d'archéologie préventive induites par les grands chantiers d'aménagements urbains. La loi du 17 janvier 2001 leur apporte un cadre juridique et crée l'Institut national pour la recherche préventive (INRAP) pour réunir les équipes nécessaires.

$\mathrm{Au}$ sein de la Caisse nationale des monuments historiques et des sites, devenue en 2000 le Centre des monuments nationaux (CMN), des « administrateurs » sont désignés en 1979 pour organiser l'accueil du public dans les meilleures conditions dans les châteaux de Chambord et de Maisons-Laffitte et dans l'abbaye du Mont-Saint-Michel ; trois autres sont chargés en 1990-1991 des châteaux d'Azay-le-Rideau, du Haut-Koenigsbourg et d'Angers et du château comtal à Carcassonne. On trouve parmi eux des profils variés d'architecte, de conservateur ou de gestionnaire culturel.

En 1996, l'ensemble des archives intermédiaires des services de l'Archéologie et des Monuments historiques, y compris la mise en place et le suivi des espaces protégés et les archives de la commission des Monuments historiques depuis 1837, est réuni dans la nouvelle médiathèque de l'Architecture et du Patrimoine.

\section{4-2015 : la décentralisation de l'Inventaire et la réforme des Monuments historiques}

37 La réforme - à partir de la loi du 13 août 2004 relative aux libertés et responsabilités locales - de la maîtrise d'ouvrage et de la maîtrise d'œuvre a modifié les conditions d'intervention de l'État sur les projets de restauration : l'initiative des travaux appartient désormais au propriétaire du monument, auquel l'État a restitué la maîtrise d'ouvrage ; les services de l'État recentrent leur action sur les édifices qui lui appartiennent, ce qui représente à peine $4 \%$ de l'ensemble des monuments classés mais correspond à des monuments majeurs : grottes ornées, cathédrales, anciens châteaux royaux et impériaux, édifices abritant les autorités gouvernementales actuelles... En plus de la maitrise d'ouvrage sur ces édifices, les services déconcentrés sont appelés à poursuivre leurs efforts pour les monuments n'appartenant pas à l'État en termes de vigilance sanitaire et de "contrôle scientifique et technique »: ils organisent, avec l'appui de l'inspection, l'instruction des demandes d'autorisation de travaux, et contrôlent à ce titre les qualifications des architectes retenus par les propriétaires.

Pour pouvoir restaurer un monument classé n'appartenant pas à l'État, les qualifications requises portent d'une part sur le diplôme (il est nécessaire d'avoir un diplôme de spécialisation et d'approfondissement mention "architecture et patrimoine " ou équivalent) et l'expérience (il faut pouvoir justifier d'une activité régulière dans le domaine de la restauration du bâti dans les dix ans qui précèdent l'année du concours) et 
d'autre part sur les références propres à l'opération - ces références tiennent compte des caractéristiques et de la complexité du projet envisagé. Les architectes en chef des monuments historiques sont qualifiés d'office relativement au diplôme et à l'expérience acquise ; ils doivent seulement justifier de références propres à l'opération. Ils sont seuls à pouvoir être chargés des monuments historiques appartenant à l'État.

L'École de Chaillot devient en 2004 le département-formation de la Cité de l'architecture et du patrimoine; le "diplôme de spécialisation et d'approfondissement " qu'elle propose, mention « Architecture et Patrimoine » est habilité l'année suivante.

La professionnalisation des métiers depuis 1830 au sein du service des Monuments historiques n'a rien d'un sujet clos. C'est un objectif qui évolue en fonction de la responsabilité que s'attribue l'État dans la politique de protection et de conservation du patrimoine. Il est sensible aux changements de cap et doit s'adapter aux réformes, notamment à la décentralisation. Les évaluations concernant les résultats du transfert des services de l'Inventaire général aux régions en témoignent : elles montrent les difficultés rencontrées pour convaincre les élus de recruter des conservateurs et poursuivre le travail engagé avec les mêmes exigences scientifiques. Les interrogations suscitées par la récente réforme des Monuments historiques pour les prochains recrutements d'architectes en chef des monuments historiques en sont aussi la preuve: on pouvait craindre qu'aucun candidat ne soit plus intéressé pour préparer ce concours long et difficile, les architectes en chef des monuments historiques n'étant plus chargés désormais d'une circonscription territoriale, mais seulement des monuments appartenant à l'État, et devant répondre à des appels à candidature pour les opérations sur les monuments dont il n'est pas propriétaire. L'organisation en 2016 d'un nouveau concours, après dix ans sans concours, et le nombre des candidats, montrent que la réforme n'a pas diminué la motivation de ceux qui aspirent à intervenir sur les monuments qui appartiennent à l'État.

Il reste à espérer que d'autres concours suivront selon le rythme de renouvellement nécessaire. Il faut aussi souhaiter que les métiers patrimoniaux, qui sont encore à ce jour à la recherche d'un statut, malgré leurs efforts de professionnalisation, notamment les conservateurs des Antiquités et Objets d'art, voient aboutir la réflexion rapidement.

\section{NOTES}

1. - BARBICHE, Bernard. «Henri IV et la surintendance des bâtiments». Bulletin Monumental, vol. 142, 1984, $\mathrm{n}^{\circ} 1$, p. 19-39.

2. - POULOT, Dominique. Une histoire des musées de France, $\mathrm{XVIII}{ }^{e}-\mathrm{XX}{ }^{e}$ siècle. Paris : La Découverte, 2005.

3. - FAVIER, Jean (dir.). La pratique archivistique française. Paris : Archives nationales, 2008.

4. - Voir le site: http://cths.fr/an/prosopo.php?id=1145 [consulté le 08/11/2018].

5. - MASSARY, Xavier de, COSTE, Georges, MELOT, Michel, VERDIER, Hélène. Principes, méthodes et conduite de l'inventaire. Paris : Éditions du patrimoine, 2001.

6. - HUGO, Victor. Pamphlets pour la sauvegarde du patrimoine. Apt : l'Archange Minotaure, 2006. 
7. - Voir le site : http://cths.fr/an/prosopo.php?id=818 [consulté le 08/11/2018].

8. - GUIZOT, François. Rapport au Roi et pièces. Paris : Imprimerie royale (Collection de documents inédits sur l'histoire de France), 1835.

9. - Voir le site : http://cths.fr/an/prosopo.php?id=65 [consulté le 08/11/2018].

10. - Voir le site : http://cths.fr/an/prosopo.php?id=66 [consulté le 08/11/2018].

11. - Voir le site : http://cths.fr/an/societe.php?id=1595\&proso=y [consulté le 08/11/2018].

12. - Voir le site : http://cths.fr/an/prosopo.php?id=3 [consulté le 08/11/2018].

13. - Voir le site : http://cths.fr/an/prosopo.php?id=109416\# [consulté le 08/11/2018].

14. - Voir le site : http://cths.fr/an/prosopo.php?id=109309 [consulté le 08/11/2018].

15. - Probablement Alphonse (Amaranthe Dugommier) Denis (1794-1876), député du Var. Voir les sites : http://www2.assemblee-nationale.fr/sycomore/fiche/\%28num_dept\%29/17494; http:// cths.fr/an/prosopo.php?id=102191; $\quad$ http://mediatheque.ville-hyeres.fr/index.php? option=com_content\&view=article\&id=327:lhomme-qui-a-lance-hyeres-alphonse-

denis\&Itemid=97 [consultés le 08/11/2018].

16. - Voir le site : http://cths.fr/an/prosopo.php?id=104889 [consulté le 08/11/2018].

17. - Voir le site $:$ http://cths.fr/an/prosopo.php?id=67 [consulté le 08/11/2018].

18. - Voir le site: http://cths.fr/hi/historique.php [consulté le 08/11/2018], et: LEROY, Rodolphe, thèse d'École des chartes, 2001, voir le site : http://theses.enc.sorbonne.fr/2001/leroy [consulté le 08/11/2018].

19. - LENIAUD, Jean-Michel. Les cathédrales au XIXe siècle: étude du service des Édifices diocésains. Paris: Caisse nationale des monuments historiques et des sites/Economica, 1993, avec le Répertoire biographique des architectes diocésains, École nationale des chartes, édition mise en ligne, 2015, voir le site : http://elec.enc.sorbonne.fr/architectes/ [consulté le 08/11/2018].

20. - VIOLLET-LE-DUC, Eugène Emmanuel. Dictionnaire raisonné de l'architecture française du $\mathrm{XI}^{e}$ au $\mathrm{XVI}^{e}$ siècle. Paris : B. Bance puis A. Morel, 1854-1868.

21. - Voir les sites : http://elec.enc.sorbonne.fr/architectes/65; http://cths.fr/an/prosopo.php? $\mathrm{id}=816$ [consultés le 08/11/2018].

22. - BERCÉ, Françoise (dir.). Les concours des monuments historiques de 1893 à 1979. Catalogue d'exposition. Paris: Caisse nationale des monuments historiques et des sites, 1981; Ead. «Formation et recrutement des architectes en chef depuis 1830 ». Monuments historiques, $\mathrm{n}^{\circ} 113$, janvier-février 1981, p. 10-22.

23. - Voir le site : http://cths.fr/an/prosopo.php?id=110008 [consulté le 08/11/2018].

24. - Voir le site : http://elec.enc.sorbonne.fr/architectes/66 [consulté le 08/11/2018].

25. - Voir le site : http://cths.fr/an/prosopo.php?id=115117 [consulté le 08/11/2018], et : KAGAN, Judith. "Des antiquités et objets d'art au patrimoine mobilier». Dans PALOUZIÉ, Hélène (dir.). Icônes et idoles. Arles : Actes Sud, 2008, p. 23-39.

26. - AUDUC, Arlette. "Quand les monuments construisaient la nation; le service des monuments historiques de 1830 à 1940 ». Paris: Comité d'histoire du ministère de la Culture, 2008 ; BERCÉ, Françoise. «L'œuvre de Paul Léon ». Dans POIRRIER, Philippe et VADELORGE, Loïc (dir). Pour une histoire des politiques du patrimoine. Paris : Comité d'histoire du ministère de la Culture, 2003.

27. - CONTENAY, Florence, MOUTON, Benjamin, PÉROUSE de MONTCLOS, Jean-Marie. L'École de Chaillot : une aventure des savoirs et des pratiques. Paris : Éd. des Cendres/Cité de l'Architecture et du Patrimoine, 2012.

28. - SOULIER, Philippe. "Soixante ans d'organisation de l'archéologie en France». Dans POIRRIER, P., VADELORGE, L. Op. cit., p. 429-450 ; MONTAGNE, Aurélie. L'Archéologie et l'État en France de 1941 à nos jours; premier cadre de l'archéologie: les directeurs des Antiquités. Mémoire de DEAC, EHESS, Paris : 2004-2005.

29. - PRESCHEZ, Philippe. "L'architecte des bâtiments de France ». La Pierre d'Angle, nos 21-22, octobre 1997. 
30. - BALSAMO, Isabelle. « André Chastel et l'aventure de l'Inventaire ». Dans NORA, Pierre (dir.). Science et Conscience du patrimoine. Actes des Entretiens du Patrimoine. Paris : Fayard/Éd. du patrimoine, 1997, p. 257-267.

31. - MAGNIEN, Aline. "Comment s'est développé l'Inventaire général des monuments et des richesses de la France ». La revue administrative, $n^{\circ} 303$, mai-juin 1998, p. 426-434; n 304, juilletaoût 1998, p. 543-550.

32. - LAURENT, Xavier. "Grandeur et misère du patrimoine d'André Malraux à Jacques Duhamel». Paris : Comité d'histoire/École nationale des chartes/La Documentation française, 2003.

33. - CHIVA, Isac. «Le patrimoine ethnologique: l'exemple de la France». Encyclopaedia Universalis, vol. 24, p. 229-241; TORNATORE, Jean-Louis. «La difficile politisation du patrimoine ethnologique ». Terrain, 2004, p.149-160; HOTTIN, Christian. "L'ethnologie, un métier du patrimoine? Réflexions autour de la question du patrimoine culturel immatériel », In Situ [En ligne], 30|2016, mis en ligne le 03 août 2017, consulté le 08 novembre 2018. URL: http:// journals.openedition.org/insitu/13633; DOI : 10.4000/insitu.13633.

34. - NORA, Pierre. «L'explosion du patrimoine ». Patrimoines, $\mathrm{n}^{\circ} 2,2006$.

\section{RÉSUMÉS}

On se fixe pour objectif de traiter dans cet article de la professionnalisation des différents métiers liés aux monuments historiques. Après un rappel consacré à l'entretien des bâtiments royaux sous l'Ancien Régime, l'attention se concentre sur la période contemporaine, depuis la Révolution jusqu'à nos jours. Les grandes étapes de cette professionnalisation sont successivement évoquées, depuis la création d'un poste d'inspecteur des monuments historiques sous la monarchie de Juillet jusqu'aux plus récentes réformes de l'administration. Cette progressive structuration concerne aussi bien les métiers de conservateur que d'architecte et est mise en relation avec le développement des autres professions concourant à la sauvegarde du patrimoine : archéologues, conservateurs et chercheurs de l'Inventaire général. Une attention particulière est apportée à la mise en place de modes de sélection appropriés (avec le développement des concours, en remplacement de la cooptation, à partir de 1884 pour les architectes diocésains et dès 1894 pour les architectes en chef des monuments historiques). Ce processus de sélection s'accompagne de la mise en place de formations spécifiques : l'École de Chaillot en 1887 pour les architectes, et, un siècle plus tard, l'École nationale du patrimoine pour les conservateurs.

The aim of this article is to examine the professionalisation of the different jobs associated with the protection of historic monuments in France. A brief introduction serves as a reminder of how royal buildings were constructed and maintained under the Ancien Régime, but the article then goes on to focus on the contemporary period since the French Revolution. The major steps in this process of professionalisation are covered, from the early creation of the post of historic monuments inspector under the July Monarchy during the 1830s, up to and including the most recent administrative reforms. This progressive structuration of the professional competences required concerns the jobs both of heritage curators and architects and is associated with other professions involved in heritage studies and conservation, such as archaeologists and curators and researchers at the national inventory service. In this examination of how jobs were given professional status, particular attention is paid to the establishment of appropriate selection 
methods, with the development of examinations gradually replacing co-optation, from 1884 for diocesan architects and from 1894 for chief architects of historic monuments. These new selection procedures were accompanied by the creation of specific training programmes, at the Ecole de Chaillot, for architects, from 1887, and, a century later, for curators, the Ecole nationale du Patrimoine, the National Heritage school.

INDEX

Mots-clés : patrimoine, monuments historiques, architecte, formation

Keywords : heritage, historic monuments, architect, training

\section{AUTEUR}

\section{MARIE-ANNE SIRE}

Inspecteur général des monuments historiques, direction générale des Patrimoines, ministère de la Culture marie-anne.sire@culture.gouv.fr 